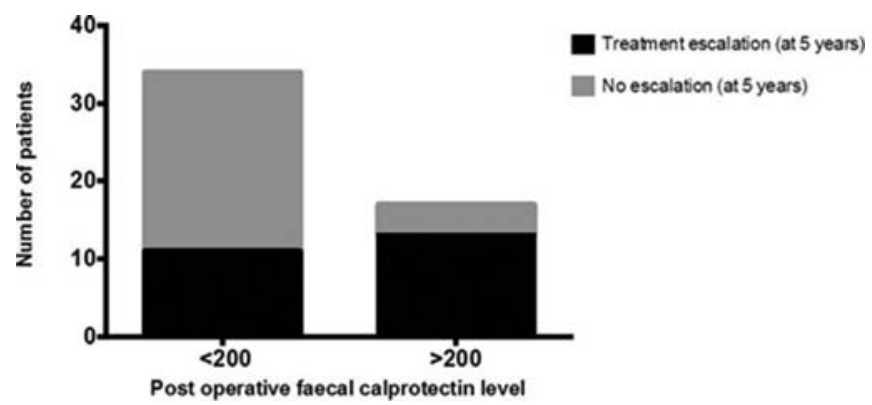

Abstract PWE-095 Figure 1

FC in post-operative Crohn's. Patients were included in the study if they had been followed up for at least 5 years after the initial FC was recorded. Case notes were reviewed retrospectively and information on the need for escalation of medical therapy or for further surgery for disease recurrence was recorded. A FC of more than 200 was taken as the cut-off value for evidence of active inflammation. Statistical analysis was performed using Prism 6 (GraphPad Software, San Diego, USA).

Results 17 patients had a FC $\geq 200$ (median 751, IQR 593916). Of these 13 required either escalation of medical treatment and/or further surgery over the 5 year follow-up period. 34 patients had a FC $<200$ (median 18, IQR 4-71). Of these 11 required treatment escalation and/or surgery. A FC of $\geq 200$ correlated significantly with need for escalation of medical treatment and/or further surgery over a 5 year follow up (p 0.003). Conclusion A FC of less than 200 predicts a better prognosis in patients with post-op Crohn's disease over a prolonged (5 year) period and could therefore potentially be used to stratify treatment and target early intervention.

Disclosure of Interest None Declared.

\section{PWE-096 THE IMPACT OF SURGEON SPECIFIC OUTCOME DATA ON PATIENT CHOICE}

R Harris*, J Mayberry. Gastroenterology, University Hospitals Leicester, Leicester, UK

\subsection{6/gutjnl-2014-307263.356}

Introduction Patients have a legal right to choose their own treatment and care within the modern NHS ${ }^{1}$. NHS England and the Association of Coloproctology of Great Britain and Ireland2, has recently released the mortality rates for individual colorectal surgeons for patients within the first 90 days of a planned operation undertaken for bowel cancer. With these figures now publicly accessible on the Internet, Gastroenterology physicians, in collaboration with their inflammatory bowel disease (IBD) patients, could utilise the information to assist in deciding who they would choose to perform the surgery should it be required. Methods One hundred and ten consecutive IBD patients who had stable disease, and seen within the outpatient setting, completed a questionnaire about which fictional surgeon they would choose dependent on the published mortality rates and their location within the country. The options included a fictional surgeon with the lowest mortality rate who was based furthest away (Newcastle), a fictional surgeon with the highest mortality rate who was local (Leicester) and a fictional surgeon with an average mortality rate who was based in between (Nottingham). There was an additional option of the patient allowing their fictional gastroenterologist to decide for them. Similarly, ten Gastroenterology colleagues were also questioned about this surgeon-specific outcome data.

\section{Patient choice}

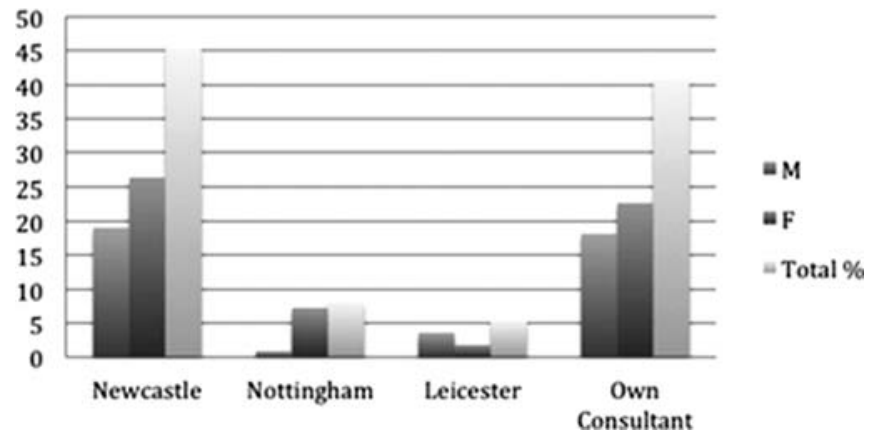

Abstract PWE-096 Figure 1

Results The majority of the 110 IBD patients chose between two options. $45 \%$ chose a fictional surgeon with the lowest mortality rate based furthest away (Newcastle) and 41\% opted for their fictional Gastroenterology consultant to decide for them. Of the 10 Gastroenterology colleagues questioned 50\% were aware that the information was now publicly accessible, 20\% were aware of where to access the information and $10 \%$ had reviewed the information online. On answering which fictional surgeon they would choose if the patient wanted their Gastroenterologist to decide for them, $40 \%$ chose the fictional surgeon with the lowest mortality rates based furthest away (Newcastle) and $20 \%$ chose the fictional surgeon with the highest mortality rates based locally (Leicester).

Conclusion The Government has proposed that the NHS allows patients to make informed choices about their own care. These results demonstrate that given that choice these IBD patients would either choose a surgeon with the lowest mortality rates, even if they were not based locally, or would allow their own Gastroenterologist to decide for them. However, the majority of the Gastroenterologists surveyed had not utilised the information on surgeon-specific outcomes. The legal consequences for gastroenterologists who choose the "less than best" option are yet to be tested in court.

\section{REFERENCES}

1 2013/2014 Choice Framework, NHS England

2 Individual surgical outcomes for England 2013, The Association of Coloproctology of Great Britain and Ireland

Disclosure of Interest None Declared.

\section{PWE-097 PATIENT KNOWLEDGE OF INFLAMMATORY BOWEL DISEASE IS NO BETTER THAN IN 1999}

${ }^{1} \mathrm{R}$ Wardle*, J Mayberry2. ${ }^{1}$ Medicine, Leicester University, Leicester, UK; ${ }^{2}$ Gastroenterology, Leicester General Hospital, Leicester, UK

\subsection{6/gutjnl-2014-307263.357}

Introduction In the UK, key professional organisations have collaborated to provide inflammatory bowel disease (IBD) Standards to be delivered by the NHS, highlighting the importance of patient education and support. ${ }^{1}$ Little literature exists however regarding the impact of these standards on patient's knowledge of their disease.

The study aim is to utilise the Crohn's and Colitis Knowledge Score (CCKNOW) to assess patient knowledge and make a comparison with results achieved in 1999.

Methods 100 outpatients with CD or UC were prospectively enrolled to complete the CCKNOW questionnaire between May and September 2013 at two Leicestershire Hospitals. 